\title{
ALLELOPATHIC ACTIVITY OF SOLVENT EXTRACTS OF RHAZYA STRICTA DECNE. AGAINST SELECTED WEEDS OF WHEAT CROP
}

\author{
ANWAR, T. ${ }^{1}$ - ILYAS, N. ${ }^{1}-$ QURESHI, R. ${ }^{{ }^{*}}-$ MUNAZIR, M. ${ }^{2}-$ KHAN, A. M. ${ }^{1}-$ ANSARI, L. ${ }^{3}-$ \\ RAHIM, B. Z. ${ }^{4}$ - ANSARI, K. A. ${ }^{5}-$ PANNI, M. K. ${ }^{6}$ \\ ${ }^{I}$ Department of Botany, Pir Mehr Ali Shah Arid Agriculture University \\ Murree Road, Shamsabad, Rawalpindi, 46300, Punjab, Pakistan \\ ${ }^{2}$ Department of Botany, Government College Women University, 51040, Sialkot, Punjab, Pakistan \\ ${ }^{3}$ Department of Forestry and Range Management, Pir Mehr Ali Shah Arid Agriculture University \\ Murree Road, Shamsabad, Rawalpindi, 46300, Punjab, Pakistan \\ ${ }^{4}$ Department of Botany, Baluchistan University, Quetta, 87500, Baluchistan, Pakistan \\ ${ }^{5}$ Department of Botany, Shah Abdul Latif University, Khairpur, 66020, Sindh, Pakistan \\ ${ }^{6}$ Bioactive Natural Products and Phytoceuticals Laboratory, Department of Horticulture, Plant \\ and Soil Building, Michigan State University, East Lansing, Michigan, 48823, U.S.A. \\ *Corresponding author \\ e-mail: rahmatullahq@yahoo.com \\ (Received $31^{\text {st }}$ May 2018; accepted $31^{\text {st }}$ Jul 2018)
}

\begin{abstract}
The experiment was designed to study allelopathic effect of Rhazya stricta Decne. against selected weeds of wheat crop by using aqueous, hexane and methanolic extracts on filter paper and soil. The germination percentage, radicle length and plumule length were observed. The results revealed that $R$. stricta methanolic extract showed the highest degree of seed germination percentage inhibition of C. album, $P$. minor and $R$. dentatus with $47 \%, 43 \%$ and $42 \%$, respectively in soil, and minimum germination for $C$. album (53\%). The same extract caused significant radical length reduction of $R$. dentatus and A. fatua measuring $51 \%$ and $50 \%$, respectively. The highest degree of inhibition in plumule length was measured for $A$. fatua and $R$. dentatus with $55 \%$ and $34 \%$, respectively by $R$. stricta methanolic extract. Based on the results, it can be concluded that $R$. stricta showed good allelopathic activity that may be used in future herbicide screening program to launch in the market.
\end{abstract}

Keywords: allelopathic effect, seed germination, radicle length, plumule length, Phalaris minor, Avena fatua, Chenopodium album, Euphorbia helioscopia, Triticum aestivum

\section{Introduction}

Weeds are regarded as a major problem in agricultural productivity of various crops globally. For controlling weeds, synthetic chemicals (weedicides) are used which possess negative effect on human health and cause environmental pollution (Dallali et al., 2017) that augments to develop alternate weed management strategies (Thi et al., 2008). Allelopathy offers an important strategy for biological weed management (Arora et al., 2015) by production and release of chemical substances from leaves, flowers, seeds, stems or roots of plants which ultimately affect growth and development of other plants (Delcour et al., 2015). The most auspicious scheme to replace synthetic herbicides is to use environmental friendly natural chemical plant products. The synthesis of biochemical based on natural plant products and management of the allelopathy towards improvement 
of crop productivity are the major areas that has gained attention of allelopathy research (Chon et al., 2005). The phytotoxic potential of these biologically active substances is widely searched by using highly advanced procedures for chemical identification (Dayan et al., 2000).

Rhazya stricta Decne., is an evergreen medicinal shrub of the family Apocynaceae distributed abundantly across Western and South Asia (Sabir et al., 2016). In Pakistan, it is commonly reported from Salt Range as well as periphery of District Khushab, Punjab, Pakistan (Qureshi et al., 2011). This plant has various ethno-medicinal values which may be attributed due to the presence of various phytochemicals in the plant. $R$. stricta invades and has deteriorated rangelands and have harmful effects on the growth and development of various plants by causing reduced seed germination (Ebid, 2016). Several alkaloids, glycosides, triterpenes, tannins and volatile compounds are present in the leaves of $R$. stricta (Khan et al., 2011). It has been reported to develop an ecological hazard such as autotoxicity to other plants due to presence of allelopathic potential (Alqarawi et al., 2018). So, it was assumed that due to presence of some chemicals $R$. stricta could be employed in biological weed management strategies. The aim of the present study was to explore the possible allelopathic potential of different leaf extracts of $R$. stricta on seed germination and seedling growth of selected weed species.

\section{Materials and methods}

\section{Collection and processing of $R$. stricta}

This research was carried out at Plant Physiology Laboratory, Department of Botany, Pir Mehr Ali Shah Arid Agriculture University, Rawalpindi (PMAS-AAUR), Pakistan. The mature leaves of $R$. stricta were collected from district Bahawalpur (73 $15^{\prime} 12^{\prime \prime} \mathrm{E}$ longitude and 29 59'55" N latitude, $159 \mathrm{~m}$ above sea level), Punjab, Pakistan and washed for several times under running tap water to remove dust following the methodology of Manimegalai et al. (2012) and Omar and Almaghrabi (2008). The same were dried in blotting paper for 4 weeks under shade at room temperature $\left(20-25^{\circ} \mathrm{C}\right)$. These were crushed using heavy duty blender (waring Lab.) to make fine powder (500 g), passed through mesh of $2 \mathrm{~mm}$ size and kept in air tight plastic zip lock bags separately at $4{ }^{\circ} \mathrm{C}$ (Anwar et al., 2013).

\section{Procurement and surface sterilization of test species seeds}

Seeds of monocotyledonous (Phalaris minor, Avena fatua) and dicotyledonous weeds (Chenopodium album, Euphorbia helioscopia and Rumex dentatus) were collected since all these are sensitive to herbicide (Shahid et al., 2006). These weeds are reported to be the most densely populated weeds of wheat crop (Qureshi et al., 2009). Wheat seeds were procured from the Barani Agricultural Research Institute (BARI), Chakwal $\left(72.7211^{\circ} \mathrm{E}\right.$ longitude and $32.9309^{\circ} \mathrm{N}$ latitude). Seeds were surface sterilized with $2 \%(\mathrm{w} / \mathrm{v})$ solution of Sodium hypochlorite $(\mathrm{NaOCl})$ for 5-8 min. After disinfection, these seeds were washed several times with distilled water (Biljana and Kragujevac, 2015; Anwar et al., 2016).

\section{Studied parameters}

Three growth parameters were used for screening allelopathic potential (Anwar et al., $2017 \mathrm{a}, \mathrm{b})$ as follows:

(a) Germination percentage 
(b) Radical length (cm)

(c) Plumule length $(\mathrm{cm})$

\section{Aqueous extract preparation}

An aliquot (10 g) of dried leaf powder of $R$. stricta was soaked in $100 \mathrm{ml}$ distilled water in a flask and agitated at room temperature $\left(20-25^{\circ} \mathrm{C}\right)$ for $24 \mathrm{~h}$ on an orbital shaker $(160 \mathrm{rpm})$. The extract was strained through muslin cloth and finally filtered through Whatman filter paper No. 1. Aqueous extract was obtained as filtrate of the mixture and final volume was attuned to $100 \mathrm{ml}$ giving $10 \%$ water extract. This stock solution was then diluted with distilled water to prepare the different concentration of the extract i.e. $\mathrm{T}_{1}(100 \%), \mathrm{T}_{2}(75 \%), \mathrm{T}_{3}(50 \%)$ after Sahu and Devkota (2013). Distilled water $(0 \%)$ was used as control and indicated as $\mathrm{T}_{4}$. The extract was stored at $4{ }^{\circ} \mathrm{C}$ in pre-disinfected flasks. To evade adulteration and forthcoming chemical modifications, the extracts were ensured to be used within 3-4 days (Anwar et al., 2017 a, b).

\section{Hexane extract preparation}

An aliquot (300 g) of dried leaf powder of $R$. stricta was mixed with hexane $(2 \mathrm{~L})$ repeated three times for $6 \mathrm{~h}$ on shaker. This extract was filtered, concentrated and dried over a rotary evaporator in pre-weighed flask. The obtained residue was $6.14 \mathrm{~g}$. The stock solution was then diluted with hexane to prepare three concentrations i.e. $\mathrm{T}_{1}$ (100\%), $\mathrm{T}_{2}(75 \%), \mathrm{T}_{3}(50 \%)$ and $\mathrm{T}_{4}\left(0 \%\right.$ control). These stored at $4{ }^{\circ} \mathrm{C}$ in pre-sterilized flasks (Sahu and Devkota, 2013).

\section{Methanolic extract preparation}

The defatted marc (after hexane extraction), was again placed on shaker with methanol $(2 \mathrm{~L})$ repeated three time for $6 \mathrm{~h}$ on shaker. This extract was filtered, concentrated, and dried over a rotary evaporator in pre-weighed flask. The obtained residue was $25.29 \mathrm{~g}$. The stock solution was than diluted with methanol to prepare three concentrations i.e. $\mathrm{T}_{1}(100 \%), \mathrm{T}_{2}(75 \%), \mathrm{T}_{3}(50 \%)$ and $\mathrm{T}_{4}(0 \%$ control). These stored at $4{ }^{\circ} \mathrm{C}$ in pre-sterilized flasks (Sahu and Devkota, 2013).

\section{Bioassay techniques}

An aliquot $(15 \mathrm{ml})$ of $R$. stricta extract was added to $25 \mathrm{~g}$ soil per Petri dish and $5 \mathrm{ml}$ extract was added to filter paper in per Petri dish; while distilled water, hexane and methanol used as control in aqueous, hexane and methanolic extracts, respectively. Ten seeds of selected test species were sown in per Petri dish. Each treatment was replicated for five times. The Petri dishes were wrapped with squash tape, enclosed with Aluminium foil and incubated in the growth chamber (NTS Model MI-25S) at room temperature $\left(20-25^{\circ} \mathrm{C}\right)$ for 15 days. The data on germination percentage, radical length $(\mathrm{cm})$ and plumule length $(\mathrm{cm})$ were recorded and calculated of each test species by comparing with control (Khan et al., 2008).

\section{Statistical analysis}

A completely randomized design (CRD) was used for the experiment. The statistical analysis was done by using STATISTIX 9 and means were separated by using Fisher's protected LSD test (Nekonam et al., 2014). 


\section{Results}

\section{Allelopathic potential of $R$. Stricta aqueous extract}

\section{Germination percentage}

The results revealed that $R$. stricta aqueous extract significantly inhibited germination of C. album (35\%), followed by R. dentatus (32\%) and P. minor (31\%) on filter paper (Table 1); whereas, no significant effect on germination percentage was recorded for T. aestivum, A. fatua and E. helioscopia. On the soil, $R$. stricta aqueous extract also significantly inhibited seed germination of $R$. dentatus $(50 \%)$, followed by C. album (45\%) and P. minor (47\%) compared to control (Tables 1 and 2). In the case of filter paper and soil, minimum germination percentage was noted for $R$. dentatus (50\%) and C. album (55\%), respectively. It has been noted that maximum germination percentage reduction of the $C$. album, $R$. dentatus and $P$. minor was accomplished at 100\% concentration revealing concentration dependent (Fig. 1a).

Table 1. Allelopathic effect of R. stricta leaf aqueous extract (AE) on germination percentage (\%) of test species on filter paper

\begin{tabular}{c|c|c|c|c|c|c}
\hline \multirow{2}{*}{ Treatments } & \multicolumn{6}{|c}{ Test species } \\
\cline { 2 - 7 } & T. aestivum & A. fatua & R. dentatus & P. minor & E. helioscopia & C. album \\
\hline $\mathrm{T}_{1 \mathrm{AE}}$ & $86^{\mathrm{a}}$ & $81^{\mathrm{a}}$ & $55^{\mathrm{c}}$ & $59^{\mathrm{c}}$ & $88^{\mathrm{a}}$ & $58^{\mathrm{c}}$ \\
$\mathrm{T}_{2 \mathrm{AE}}$ & $88^{\mathrm{a}}$ & $80^{\mathrm{a}}$ & $59^{\mathrm{c}}$ & $61^{\mathrm{c}}$ & $90^{\mathrm{a}}$ & $67^{\mathrm{c}}$ \\
$\mathrm{T}_{3 \mathrm{AE}}$ & $89^{\mathrm{a}}$ & $82^{\mathrm{a}}$ & $70^{\mathrm{b}}$ & $74^{\mathrm{b}}$ & $91^{\mathrm{a}}$ & $78^{\mathrm{b}}$ \\
$\mathrm{T}_{4 \mathrm{AE}}$ & $90^{\mathrm{a}}$ & $83^{\mathrm{a}}$ & $81^{\mathrm{a}}$ & $85^{\mathrm{a}}$ & $92^{\mathrm{a}}$ & $89^{\mathrm{a}}$ \\
$\mathrm{LSD}$ & 14.722 & 18.56 & 16.974 & 17.874 & 17.529 & 17.504 \\
F-value & $27.64^{*}$ & $25.08^{*}$ & $13.29^{*}$ & $14.98^{*}$ & $21.73^{*}$ & $14.97^{*}$ \\
\hline
\end{tabular}

Means followed by different letters within one column differ significantly at $(P<5 \%)$

*Significant at $(P<1 \%)$

Table 2. Allelopathic effect of $R$. stricta leaf aqueous extract (AE) on germination percentage (\%) of test species on soil

\begin{tabular}{c|c|c|c|c|c|c}
\hline \multirow{2}{*}{ Treatments } & \multicolumn{6}{|c}{ Test species } \\
\cline { 2 - 7 } & T. aestivum & A. fatua & R. dentatus & P. minor & E. helioscopia & C. album \\
\hline $\mathrm{T}_{1 \mathrm{AE}}$ & $87^{\mathrm{a}}$ & $80^{\mathrm{a}}$ & $50^{\mathrm{c}}$ & $53^{\mathrm{b}}$ & $89^{\mathrm{a}}$ & $55^{\mathrm{c}}$ \\
$\mathrm{T}_{2 \mathrm{AE}}$ & $89^{\mathrm{a}}$ & $82^{\mathrm{a}}$ & $56^{\mathrm{c}}$ & $58^{\mathrm{b}}$ & $91^{\mathrm{a}}$ & $64^{\mathrm{a}}$ \\
$\mathrm{T}_{3 \mathrm{AE}}$ & $90^{\mathrm{a}}$ & $83^{\mathrm{a}}$ & $67^{\mathrm{b}}$ & $71^{\mathrm{b}}$ & $92^{\mathrm{a}}$ & $75^{\mathrm{b}}$ \\
$\mathrm{T}_{4 \mathrm{AE}}$ & $91^{\mathrm{a}}$ & $84^{\mathrm{a}}$ & $82^{\mathrm{a}}$ & $86^{\mathrm{a}}$ & $93^{\mathrm{a}}$ & $90^{\mathrm{a}}$ \\
$\mathrm{LSD}$ & 15.722 & 19.46 & 15.774 & 14.474 & 16.529 & 17.967 \\
F-value & $26.64^{*}$ & $24.07^{*}$ & $12.39^{*}$ & $15.68^{*}$ & $22.73^{*}$ & $31.90^{*}$ \\
\hline
\end{tabular}

Means followed by different letters within one column differ significantly at $(P<5 \%)$

*Significant at $(P<1 \%)$

\section{Radical length}

The data revealed that the highest radical length inhibition activity was exhibited by $R$. dentatus (44\%) and A. fatua (40\%) in R. stricta aqueous extract on filter paper. 
Likewise, extract on soil caused significant radical length reduction of $R$. dentatus (48\%) followed by A. fatua (45\%) as compared to control. The data suggested that radical length of $C$. album, $P$. minor, E. helioscopia and $T$. aestivum remained unaffected. The results also illustrated that minimum radical length was noted for $A$. fatua $(56 \%)$ and $R$. dentatus (52\%) on filter paper and soil, respectively. The final data concluded that maximum radical length $(97 \%)$ was noted for C. album, P. minor, E. helioscopia and T. aestivum (Tables 3 and 4). The results revealed that allelopathic inhibitory effect was concentration dependent for $R$. dentatus and A. fatua (Fig. 1b).

Table 3. Allelopathic effect of $R$. stricta aqueous extract (AE) on radical length (cm) of test species on filter paper

\begin{tabular}{c|c|c|c|c|c|c}
\hline \multirow{2}{*}{ Treatments } & \multicolumn{6}{|c}{ Test species } \\
\cline { 2 - 7 } & T. aestivum & A. fatua & R. dentatus & P. minor & E. helioscopia & C. album \\
\hline $\mathrm{T}_{1 \mathrm{AE}}$ & $9.03^{\mathrm{a}}$ & $4.99^{\mathrm{c}}$ & $5.08^{\mathrm{d}}$ & $8.11^{\mathrm{a}}$ & $9.13^{\mathrm{a}}$ & $8.12^{\mathrm{a}}$ \\
$\mathrm{T}_{2 \mathrm{AE}}$ & $9.06^{\mathrm{a}}$ & $5.87^{\mathrm{c}}$ & $6.82^{\mathrm{c}}$ & $8.14^{\mathrm{a}}$ & $9.22^{\mathrm{a}}$ & $8.19^{\mathrm{a}}$ \\
$\mathrm{T}_{3 \mathrm{AE}}$ & $9.08^{\mathrm{a}}$ & $7.13^{\mathrm{b}}$ & $8.04^{\mathrm{b}}$ & $8.18^{\mathrm{a}}$ & $9.37^{\mathrm{a}}$ & $8.21^{\mathrm{a}}$ \\
$\mathrm{T}_{4 \mathrm{AE}}$ & $9.14^{\mathrm{a}}$ & $8.35^{\mathrm{a}}$ & $9.14^{\mathrm{a}}$ & $8.20^{\mathrm{a}}$ & $9.41^{\mathrm{a}}$ & $8.24^{\mathrm{a}}$ \\
$\mathrm{LSD}$ & 4.2679 & 0.9692 & 0.7136 & 2.4388 & 1.403 & 0.9741 \\
F-value & $20.22^{*}$ & $486.76^{*}$ & $278.70^{*}$ & $58.00^{*}$ & $55.71^{*}$ & $161.39^{*}$ \\
\hline
\end{tabular}

Means followed by different letters within one column differ significantly at $(P<5 \%)$

* Significant at $(P<1 \%)$

Table 4. Allelopathic effect of $R$. stricta aqueous extract (AE) on radical length ( $\mathrm{cm}$ ) of test species on soil

\begin{tabular}{c|c|c|c|c|c|c}
\hline \multirow{2}{*}{ Treatments } & \multicolumn{6}{|c}{ Test species } \\
\cline { 2 - 7 } & T. aestivum & A. fatua & R. dentatus & P. minor & E. helioscopia & C. album \\
\hline $\mathrm{T}_{1 \mathrm{~A}}$ & $9.12^{\mathrm{a}}$ & $4.75^{\mathrm{c}}$ & $4.82^{\mathrm{c}}$ & $8.18^{\mathrm{a}}$ & $9.22^{\mathrm{a}}$ & $8.27^{\mathrm{a}}$ \\
$\mathrm{T}_{2 \mathrm{~A}}^{\mathrm{a}}$ & $9.26^{\mathrm{a}}$ & $5.02^{\mathrm{c}}$ & $6.55^{\mathrm{b}}$ & $8.23^{\mathrm{a}}$ & $9.41^{\mathrm{a}}$ & $8.31^{\mathrm{a}}$ \\
$\mathrm{T}_{3 \mathrm{AE}}$ & $9.32^{\mathrm{a}}$ & $6.99^{\mathrm{b}}$ & $7.09^{\mathrm{b}}$ & $8.40^{\mathrm{a}}$ & $9.55^{\mathrm{a}}$ & $8.46^{\mathrm{a}}$ \\
$\mathrm{T}_{4 \mathrm{AE}}$ & $9.38^{\mathrm{a}}$ & $8.65^{\mathrm{a}}$ & $9.35^{\mathrm{a}}$ & $8.49^{\mathrm{a}}$ & $9.67^{\mathrm{a}}$ & $8.59^{\mathrm{a}}$ \\
$\mathrm{LSD}$ & 1.1360 & 1.540 & 0.999 & 1.1125 & 1.0662 & 1.320 \\
F-value & $127.48^{*}$ & $45.47^{*}$ & $66.33^{*}$ & $111.84^{*}$ & $95.32^{*}$ & $56.72^{*}$ \\
\hline
\end{tabular}

Means followed by different letters within one column differ significantly at $(P<5 \%)$

*Significant at $(P<1 \%)$

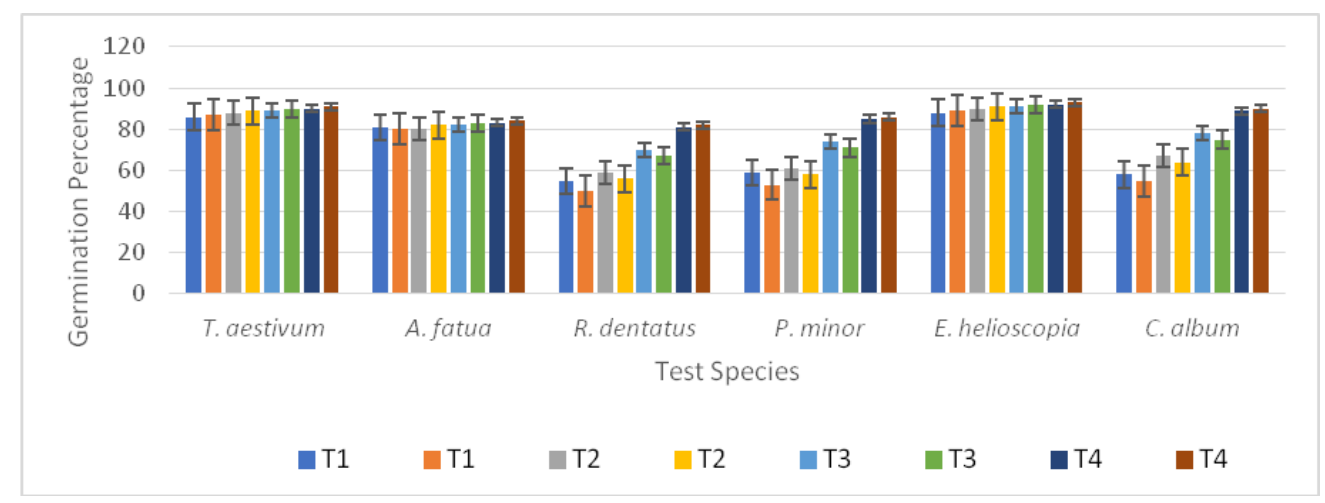




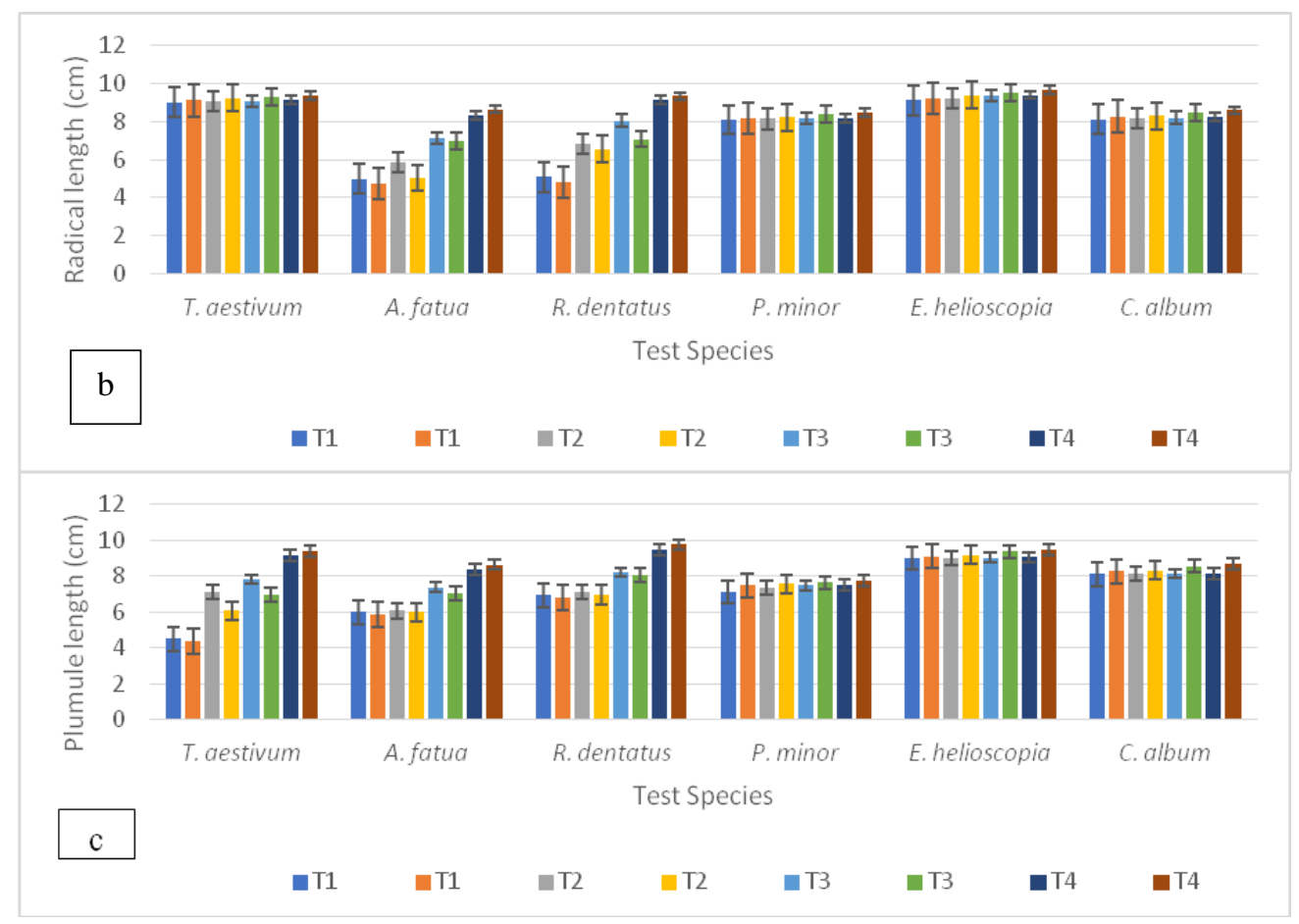

Figure 1. Allelopathic potential of R. stricta aqueous extract against test species on filter paper $(F P)$ and soil $(S)$ on: (a) germination percentage $(b)$ radical length (c) plumule length

\section{Plumule length}

The experimental results of the current study indicated that aqueous extract of $R$. stricta significantly inhibited the plumule length of T. aestivum (51\%), A. fatua (28\%) and $R$. dentatus $(27 \%)$ as compared to control, on filter paper. Interestingly, there was no significant effect on plumule elongation of $P$. minor, E. helioscopia and C. album. The statistical study recommended that $R$. stricta aqueous extract significantly inhibited plumule length of T. aestivum (53\%), A. fatua (32\%) and $R$. dentatus (30\%) in soil. The statistical data concluded that minimum plumule length was noted for $T$. aestivum i.e. $49 \%$ and $47 \%$ on filter paper and soil, respectively (Tables 5 and 6 ). The statistical figures also proposed that maximum plumule length (96\%) was noted for P. minor, E. helioscopia and C. album (Fig. 1c).

Table 5. Allelopathic effect of $R$. stricta aqueous extract (AE) on plumule length ( $\mathrm{cm}$ ) of test species on filter paper

\begin{tabular}{c|c|c|c|c|c|c}
\hline \multirow{2}{*}{ Treatments } & \multicolumn{6}{|c}{ Test species } \\
\cline { 2 - 7 } & T. aestivum & A. fatua & R. dentatus & P. minor & E. helioscopia & C. album \\
\hline $\mathrm{T}_{1 \mathrm{AE}}$ & $4.49^{\mathrm{c}}$ & $5.99^{\mathrm{c}}$ & $6.93^{\mathrm{c}}$ & $7.12^{\mathrm{a}}$ & $9.01^{\mathrm{a}}$ & $8.11^{\mathrm{a}}$ \\
$\mathrm{T}_{2 \mathrm{AE}}$ & $7.12^{\mathrm{b}}$ & $6.06^{\mathrm{c}}$ & $7.13^{\mathrm{c}}$ & $7.36^{\mathrm{a}}$ & $9.02^{\mathrm{a}}$ & $8.12^{\mathrm{a}}$ \\
$\mathrm{T}_{3 \mathrm{AE}}$ & $7.81^{\mathrm{b}}$ & $7.39^{\mathrm{b}}$ & $8.24^{\mathrm{b}}$ & $7.48^{\mathrm{a}}$ & $9.04^{\mathrm{a}}$ & $8.13^{\mathrm{a}}$ \\
$\mathrm{T}_{4 \mathrm{AE}}$ & $9.14^{\mathrm{a}}$ & $8.39^{\mathrm{a}}$ & $9.46^{\mathrm{a}}$ & $7.52^{\mathrm{a}}$ & $9.06^{\mathrm{a}}$ & $8.14^{\mathrm{a}}$ \\
$\mathrm{LSD}$ & 3.7846 & 1.2971 & 0.9640 & 0.8718 & $0.0182^{*}$ & 0.6156 \\
F-value & $8.44^{* *}$ & $46.74^{*}$ & $57.31^{*}$ & $55.81^{*}$ & $14.11^{*}$ & $111.35^{*}$ \\
\hline
\end{tabular}

Means followed by different letters within one column differ significantly at $(P<5 \%)$

*Significant at $(P<1 \%)$ 
Table 6. Allelopathic effect of $R$. stricta aqueous extract $(A E)$ on plumule length ( $\mathrm{cm})$ of test species on soil

\begin{tabular}{c|c|c|c|c|c|c}
\hline \multirow{2}{*}{ Treatments } & \multicolumn{6}{|c}{ Test species } \\
\cline { 2 - 7 } & T. aestivum & A. fatua & R. dentatus & P. minor & E. helioscopia & C. album \\
\hline $\mathrm{T}_{1 \mathrm{AE}}$ & $4.36^{\mathrm{c}}$ & $5.86^{\mathrm{c}}$ & $6.82^{\mathrm{c}}$ & $7.48^{\mathrm{a}}$ & $9.12^{\mathrm{a}}$ & $8.26^{\mathrm{a}}$ \\
$\mathrm{T}_{2 \mathrm{AE}}$ & $6.08^{\mathrm{b}}$ & $5.99^{\mathrm{c}}$ & $6.96^{\mathrm{c}}$ & $7.56^{\mathrm{a}}$ & $9.20^{\mathrm{a}}$ & $8.32^{\mathrm{a}}$ \\
$\mathrm{T}_{3 \mathrm{AE}}$ & $6.95^{\mathrm{b}}$ & $7.06^{\mathrm{b}}$ & $8.07^{\mathrm{b}}$ & $7.63^{\mathrm{a}}$ & $9.37^{\mathrm{a}}$ & $8.57^{\mathrm{a}}$ \\
$\mathrm{T}_{4 \mathrm{AE}}$ & $9.38^{\mathrm{a}}$ & $8.65^{\mathrm{a}}$ & $9.76^{\mathrm{a}}$ & $7.72^{\mathrm{a}}$ & $9.46^{\mathrm{a}}$ & $8.69^{\mathrm{a}}$ \\
$\mathrm{LSD}$ & 2.7846 & 1.3971 & 0.9140 & 0.8618 & 0.1182 & 0.4294 \\
F-value & $6.44^{* *}$ & $45.74^{*}$ & $56.31^{*}$ & $55.91^{*}$ & $14.21^{*}$ & $196.36^{*}$ \\
\hline
\end{tabular}

Means followed by different letters within one column differ significantly at $(P<5 \%)$

* Significant at $(P<1 \%)$

\section{Allelopathic potential of $R$. stricta hexane extract}

\section{Germination percentage}

There was significant reduction of germination of $C$. album (42\%), $R$. dentatus (45\%) and $P$. minor (41\%) as compared to control on filter paper, whereas, no significant effect was observed on the germination of $T$. aestivum, A. fatua and $E$. helioscopia showing resistance to the allelopathic effect. Similarly, the highest seed germination inhibition was noted for $C$. album (51\%), P. minor (50\%) and $R$. dentatus $(51 \%)$ by incorporation of $R$. stricta hexane extract into soil. It was experiential that the maximum (97\%) germination noted for T. aestivum, A. fatua and E. helioscopia. In the present study, minimum germination was noted for $R$. dentatus $(65 \%)$ and $C$. album (49\%) on filter paper and soil, respectively (Tables 7 and 8). The statistics also recommended that allelopathic inhibitory effect was concentration dependent for $C$. album, $P$. minor and $R$. dentatus with concentration increase, suppression potential was gradually enhanced (Fig. $2 a$ ).

Table 7. Allelopathic effect of $R$. stricta leaf hexane extract (HE) on germination percentage (\%) of test species on filter paper

\begin{tabular}{c|c|c|c|c|c|c}
\hline \multirow{2}{*}{ Treatments } & \multicolumn{6}{|c}{ Test species } \\
\cline { 2 - 7 } & T. aestivum & A. fatua & R. dentatus & P. minor & E. helioscopia & C. album \\
\hline $\mathrm{T}_{1 \mathrm{HE}}$ & $86^{\mathrm{a}}$ & $81^{\mathrm{a}}$ & $55^{\mathrm{c}}$ & $59^{\mathrm{c}}$ & $88^{\mathrm{a}}$ & $58^{\mathrm{c}}$ \\
$\mathrm{T}_{2 \mathrm{HE}}$ & $88^{\mathrm{a}}$ & $80^{\mathrm{a}}$ & $59^{\mathrm{c}}$ & $61^{\mathrm{c}}$ & $90^{\mathrm{a}}$ & $67^{\mathrm{c}}$ \\
$\mathrm{T}_{3 \mathrm{HE}}$ & $89^{\mathrm{a}}$ & $82^{\mathrm{a}}$ & $70^{\mathrm{b}}$ & $74^{\mathrm{b}}$ & $91^{\mathrm{a}}$ & $78^{\mathrm{b}}$ \\
$\mathrm{T}_{4 \mathrm{HE}}$ & $90^{\mathrm{a}}$ & $83^{\mathrm{a}}$ & $81^{\mathrm{a}}$ & $85^{\mathrm{a}}$ & $92^{\mathrm{a}}$ & $89^{\mathrm{a}}$ \\
$\mathrm{LSD}$ & 14.722 & 18.56 & 16.974 & 17.874 & 17.529 & 17.504 \\
F-value & $27.64^{*}$ & $25.08^{*}$ & $13.29^{*}$ & $14.98^{*}$ & $21.73^{*}$ & $14.97^{*}$ \\
\hline
\end{tabular}

Means followed by different letters within one column differ significantly at $(P<5 \%)$

*Significant at $(P<1 \%)$ 
Table 8. Allelopathic effect of $R$. stricta leaf hexane extract (HE) on germination percentage (\%) of test species on soil

\begin{tabular}{c|c|c|c|c|c|c}
\hline \multirow{2}{*}{ Treatments } & \multicolumn{6}{|c}{ Test species } \\
\cline { 2 - 7 } & T. aestivum & A. fatua & R. dentatus & P. minor & E. helioscopia & C. album \\
\hline $\mathrm{T}_{1 \mathrm{HE}}$ & $89^{\mathrm{a}}$ & $82^{\mathrm{a}}$ & $49^{\mathrm{c}}$ & $50^{\mathrm{d}}$ & $91^{\mathrm{a}}$ & $49^{\mathrm{d}}$ \\
$\mathrm{T}_{2 \mathrm{HE}}$ & $91^{\mathrm{a}}$ & $84^{\mathrm{a}}$ & $56^{\mathrm{c}}$ & $61^{\mathrm{c}}$ & $93^{\mathrm{a}}$ & $64^{\mathrm{c}}$ \\
$\mathrm{T}_{3 \mathrm{HE}}$ & $92^{\mathrm{a}}$ & $85^{\mathrm{a}}$ & $68^{\mathrm{b}}$ & $71^{\mathrm{b}}$ & $94^{\mathrm{a}}$ & $75^{\mathrm{b}}$ \\
$\mathrm{T}_{4 \mathrm{HE}}$ & $93^{\mathrm{a}}$ & $86^{\mathrm{a}}$ & $84^{\mathrm{a}}$ & $88^{\mathrm{a}}$ & $95^{\mathrm{a}}$ & $92^{\mathrm{a}}$ \\
$\mathrm{LSD}$ & 13.588 & 17.910 & 15.07 & 13.844 & 15.480 & 15.874 \\
F-value & $13.93^{*}$ & $22.96^{*}$ & $37.71^{*}$ & $22.94^{*}$ & $54.38^{*}$ & $12.98^{*}$ \\
\hline
\end{tabular}

Means followed by different letters within one column differ significantly at $(P<5 \%)$

* Significant at $(P<1 \%)$

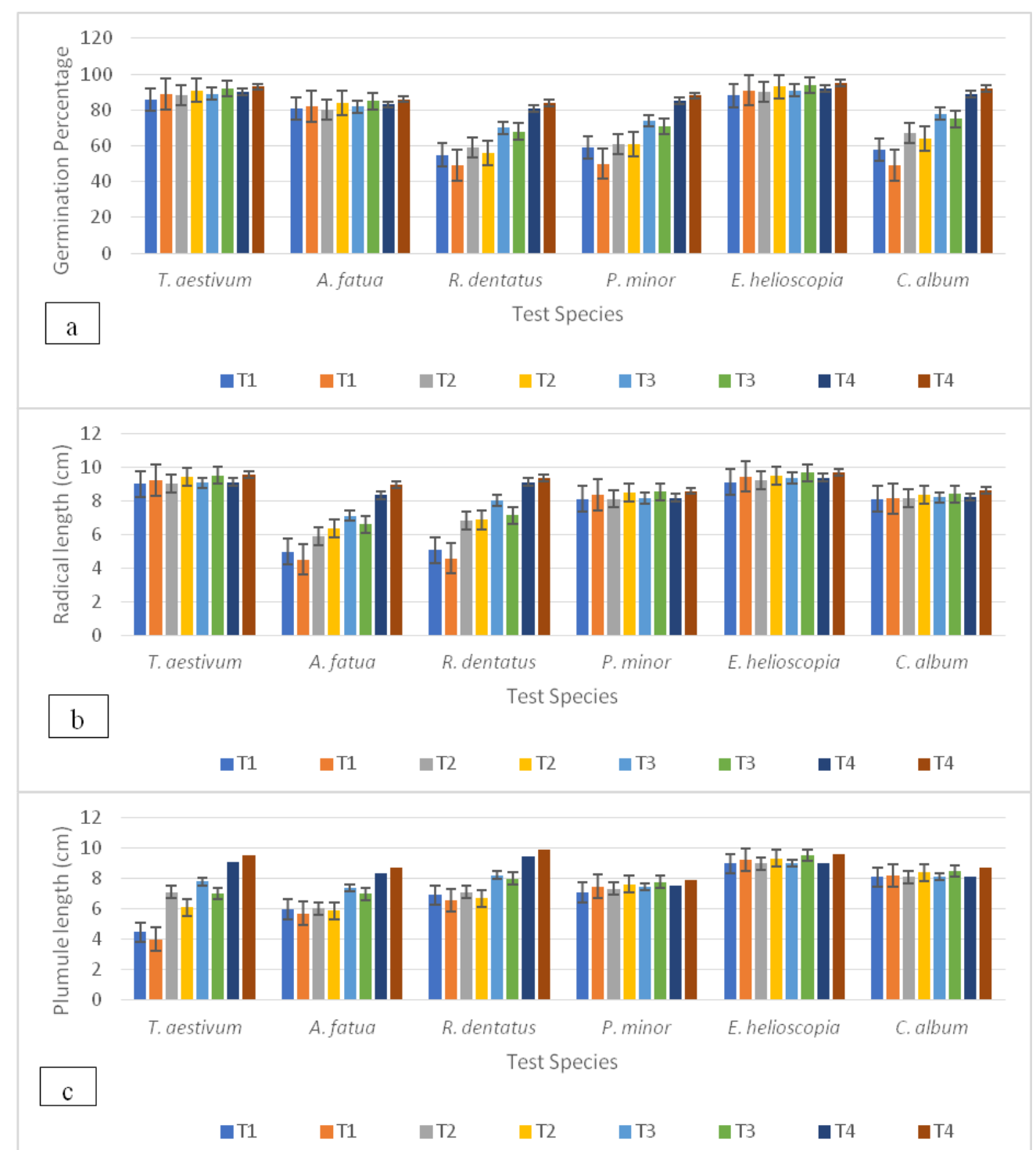

Figure 2. Allelopathic potential of $R$. stricta hexane extract against test species on filter paper $(F P)$ and soil $(S)$ on: (a) germination percentage $(b)$ radical length $(c)$ plumule length 


\section{Radical length}

The statistical data concluded that the highest radical length inhibition activity was exhibited by $R$. dentatus (44\%) and A. fatua (40\%) in $R$. stricta hexane extract on filter paper. Similarly, hexane extract on soil caused significant reduction in radical length of $R$. dentatus (51\%) and A. fatua (50\%) compared to control, while T. aestivum, P. minor, E. helioscopia and $C$. album remained unaffected. The results also illustrated minimum radical length for $R$. dentatus i.e. $46 \%$ and $49 \%$ on filter paper and soil, respectively (Tables 9 and 10). The maximum radical length (96\%) was noted for T. aestivum, $P$. minor, E. helioscopia and C. album (Fig. 2b).

Table 9. Allelopathic effect of $R$. stricta leaf hexane extract $(H E)$ on radical length $(\mathrm{cm})$ of test species on filter paper

\begin{tabular}{c|c|c|c|c|c|c}
\hline \multirow{2}{*}{ Treatments } & \multicolumn{6}{|c}{ Test species } \\
\cline { 2 - 7 } & T. aestivum & A. fatua & R. dentatus & P. minor & E. helioscopia & C. album \\
\hline $\mathrm{T}_{1 \mathrm{HE}}$ & $9.03^{\mathrm{a}}$ & $4.99^{\mathrm{c}}$ & $5.08^{\mathrm{d}}$ & $8.11^{\mathrm{a}}$ & $9.13^{\mathrm{a}}$ & $8.12^{\mathrm{a}}$ \\
$\mathrm{T}_{2 \mathrm{HE}}$ & $9.06^{\mathrm{a}}$ & $5.87^{\mathrm{c}}$ & $6.82^{\mathrm{c}}$ & $8.14^{\mathrm{a}}$ & $9.22^{\mathrm{a}}$ & $8.19^{\mathrm{a}}$ \\
$\mathrm{T}_{3 \mathrm{HE}}$ & $9.08^{\mathrm{a}}$ & $7.13^{\mathrm{b}}$ & $8.04^{\mathrm{b}}$ & $8.18^{\mathrm{a}}$ & $9.37^{\mathrm{a}}$ & $8.21^{\mathrm{a}}$ \\
$\mathrm{T}_{4 \mathrm{HE}}$ & $9.14^{\mathrm{a}}$ & $8.35^{\mathrm{a}}$ & $9.14^{\mathrm{a}}$ & $8.20^{\mathrm{a}}$ & $9.41^{\mathrm{a}}$ & $8.24^{\mathrm{a}}$ \\
$\mathrm{LSD}$ & 4.2679 & 0.9692 & 0.7136 & 2.4388 & 1.403 & 0.9741 \\
F-value & $20.22^{*}$ & $486.76^{*}$ & $278.70^{*}$ & $58.00^{*}$ & $55.71^{*}$ & $161.39^{*}$ \\
\hline
\end{tabular}

Means followed by different letters within one column differ significantly at $(P<5 \%)$

* Significant at $(P<1 \%)$

Table 10. Allelopathic effect of $R$. stricta leaf hexane extract $(H E)$ on radical length ( $\mathrm{cm}$ ) of test species on soil

\begin{tabular}{c|c|c|c|c|c|c}
\hline \multirow{2}{*}{ Treatments } & \multicolumn{6}{|c}{ Test species } \\
\cline { 2 - 7 } & T. aestivum & A. fatua & R. dentatus & P. minor & E. helioscopia & C. album \\
\hline $\mathrm{T}_{1 \mathrm{HE}}$ & $9.26^{\mathrm{a}}$ & $4.52^{\mathrm{c}}$ & $4.58^{\mathrm{c}}$ & $8.39^{\mathrm{a}}$ & $9.48^{\mathrm{a}}$ & $8.15^{\mathrm{a}}$ \\
$\mathrm{T}_{2 \mathrm{HE}}$ & $9.46^{\mathrm{a}}$ & $6.34^{\mathrm{b}}$ & $6.87^{\mathrm{b}}$ & $8.48^{\mathrm{a}}$ & $9.52^{\mathrm{a}}$ & $8.35^{\mathrm{a}}$ \\
$\mathrm{T}_{3 \mathrm{HE}}$ & $9.52^{\mathrm{a}}$ & $6.61^{\mathrm{b}}$ & $7.16^{\mathrm{b}}$ & $8.56^{\mathrm{a}}$ & $9.69^{\mathrm{a}}$ & $8.43^{\mathrm{a}}$ \\
$\mathrm{T}_{4 \mathrm{HE}}$ & $9.58^{\mathrm{a}}$ & $8.99^{\mathrm{a}}$ & $9.40^{\mathrm{a}}$ & $8.61^{\mathrm{a}}$ & $9.70^{\mathrm{a}}$ & $8.66^{\mathrm{a}}$ \\
LSD & 1.8406 & 1.3918 & 1.9912 & 1.5634 & 0.3063 & 1.4662 \\
F-value & $51.46^{*}$ & $97.97^{*}$ & $84.01^{*}$ & $76.89^{*}$ & $125.70^{*}$ & $93.12^{*}$ \\
\hline
\end{tabular}

Means followed by different letters within one column differ significantly at $(P<5 \%)$

*Significant at $(P<1 \%)$

\section{Plumule length}

The $R$. stricta hexane extract significantly inhibited the plumule length of $T$. aestivum (51\%), A. fatua (29\%) and $R$. dentatus (27\%) on filter paper. Interestingly, there was no significant effect on plumule elongation of $P$. minor, E. helioscopia and $C$. album. Likewise, $R$. stricta hexane extract significantly inhibited plumule length of $T$. aestivum (58\%), A. fatua (34\%) and R. dentatus (34\%) in soil. The statistical data concluded that minimum plumule length was noted for T. aestivum i.e. $49 \%$ and $42 \%$ on filter paper and soil, respectively (Tables 11 and 12). The statistical analysis revealed 
that maximum plumule length (95\%) was noted for $P$. minor, E. helioscopia and $C$. album (Fig. 2c).

Table 11. Allelopathic effect of $R$. stricta leaf hexane extract $(H E)$ on plumule length $(\mathrm{cm})$ of test species on filter paper

\begin{tabular}{c|c|c|c|c|c|c}
\hline \multirow{2}{*}{ Treatments } & \multicolumn{6}{|c}{ Test species } \\
\cline { 2 - 7 } & T. aestivum & A. fatua & R. dentatus & P. minor & E. helioscopia & C. album \\
\hline $\mathrm{T}_{1 \mathrm{HE}}$ & $4.49^{\mathrm{c}}$ & $5.99^{\mathrm{c}}$ & $6.93^{\mathrm{c}}$ & $7.12^{\mathrm{a}}$ & $9.01^{\mathrm{a}}$ & $8.11^{\mathrm{a}}$ \\
$\mathrm{T}_{2 \mathrm{HE}}$ & $7.12^{\mathrm{b}}$ & $6.06^{\mathrm{c}}$ & $7.13^{\mathrm{c}}$ & $7.36^{\mathrm{a}}$ & $9.02^{\mathrm{a}}$ & $8.12^{\mathrm{a}}$ \\
$\mathrm{T}_{3 \mathrm{HE}}$ & $7.81^{\mathrm{b}}$ & $7.39^{\mathrm{b}}$ & $8.24^{\mathrm{b}}$ & $7.48^{\mathrm{a}}$ & $9.04^{\mathrm{a}}$ & $8.13^{\mathrm{a}}$ \\
$\mathrm{T}_{4 \mathrm{HE}}$ & $9.14^{\mathrm{a}}$ & $8.39^{\mathrm{a}}$ & $9.46^{\mathrm{a}}$ & $7.52^{\mathrm{a}}$ & $9.06^{\mathrm{a}}$ & $8.14^{\mathrm{a}}$ \\
$\mathrm{LSD}$ & 3.7846 & 1.2971 & 0.9640 & 0.8718 & 0.0182 & 0.6156 \\
F-value & $8.44^{* *}$ & $46.74^{*}$ & $57.31^{*}$ & $55.81^{*}$ & $14.11^{*}$ & $111.35^{*}$ \\
\hline
\end{tabular}

Means followed by different letters within one column differ significantly at $(P<5 \%)$

*Significant at $(P<1 \%)$

Table 12. Allelopathic effect of $R$. stricta leaf hexane extract $(H E)$ on plumule length $(\mathrm{cm})$ of test species on soil

\begin{tabular}{c|c|c|c|c|c|c}
\hline \multirow{2}{*}{ Treatments } & \multicolumn{6}{|c}{ Test species } \\
\cline { 2 - 7 } & T. aestivum & A. fatua & R. dentatus & P. minor & E. helioscopia & C. album \\
\hline $\mathrm{T}_{1 \mathrm{HE}}$ & $4.01^{\mathrm{c}}$ & $5.72^{\mathrm{c}}$ & $6.59^{\mathrm{c}}$ & $7.51^{\mathrm{a}}$ & $9.24^{\mathrm{a}}$ & $8.21^{\mathrm{a}}$ \\
$\mathrm{T}_{2 \mathrm{HE}}$ & $6.13^{\mathrm{b}}$ & $5.90^{\mathrm{c}}$ & $6.72^{\mathrm{c}}$ & $7.65^{\mathrm{a}}$ & $9.33^{\mathrm{a}}$ & $8.42^{\mathrm{a}}$ \\
$\mathrm{T}_{3 \mathrm{HE}}$ & $7.04^{\mathrm{b}}$ & $7.01^{\mathrm{b}}$ & $8.01^{\mathrm{b}}$ & $7.79^{\mathrm{a}}$ & $9.56^{\mathrm{a}}$ & $8.51^{\mathrm{a}}$ \\
$\mathrm{T}_{4 \mathrm{HE}}$ & $9.58^{\mathrm{a}}$ & $8.77^{\mathrm{a}}$ & $9.94^{\mathrm{a}}$ & $7.89^{\mathrm{a}}$ & $9.61^{\mathrm{a}}$ & $8.71_{\mathrm{a}}$ \\
$\mathrm{LSD}$ & 3.7946 & 2.2971 & 1.9640 & 2.8718 & 4.0182 & 0.9841 \\
F-value & $9.34^{* *}$ & $41.64^{*}$ & $53.41^{*}$ & $58.71^{*}$ & $16.31^{*}$ & $246.78^{*}$ \\
\hline
\end{tabular}

Means followed by different letters within one column differ significantly at $(P<5 \%)$

*Significant at $(P<1 \%)$

\section{Allelopathic potential of $R$. stricta methanolic extract}

\section{Germination percentage}

The methanolic extract of $R$. stricta significantly inhibited germination of $P$. minor (49\%), R. dentatus (48\%) and C. album (47\%) on filter paper, whereas, no significant effect was noted on the germination of T. aestivum, A. fatua and E. helioscopia showing resistance against extract. Similarly, the same extract significantly suppressed seed germination of $C$. album and $R$. dentatus (51\% each), followed by $P$. minor (50\%) on soil. It was noted that maximum (95\%) germination was observed for T. aestivum, A. fatua and E. helioscopia. Besides, minimum germination was noted for P. minor i.e. $51 \%$ and $R$. dentatus (49\%) on filter paper and soil, respectively (Tables 13 and 14). The results revealed that germination reduction of the $C$. album, $P$. minor and $R$. dentatus was concentration dependent (Fig. 3a). 


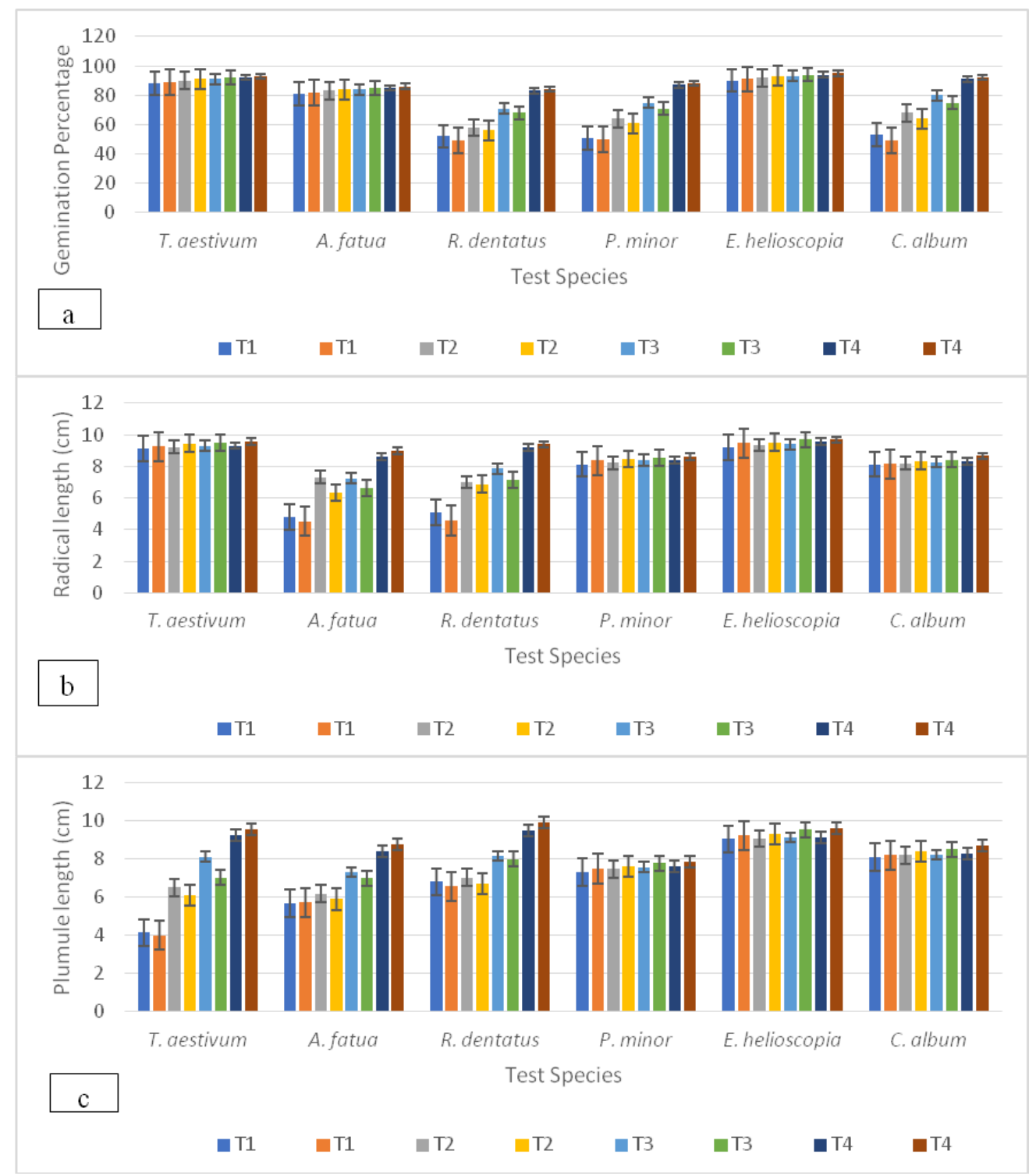

Figure 3. Allelopathic potential of R. stricta methanolic extract against test species on filter paper (FP) and soil (S) on: (a) germination percentage (b) radical length (c) plumule length

Table 13. Allelopathic effect of $R$. stricta leaf methanolic extract (ME) on germination percentage (\%) of test species on filter paper

\begin{tabular}{c|c|c|c|c|c|c}
\hline \multirow{2}{*}{ Treatments } & \multicolumn{6}{|c}{ Test species } \\
\cline { 2 - 7 } & T. aestivum & A. fatua & R. dentatus & P. minor & E. helioscopia & C. album \\
\hline $\mathrm{T}_{1 \mathrm{ME}}$ & $88^{\mathrm{a}}$ & $81^{\mathrm{a}}$ & $52^{\mathrm{c}}$ & $51^{\mathrm{d}}$ & $90^{\mathrm{a}}$ & $53^{\mathrm{d}}$ \\
$\mathrm{T}_{2 \mathrm{ME}}$ & $90^{\mathrm{a}}$ & $83^{\mathrm{a}}$ & $58^{\mathrm{c}}$ & $64^{\mathrm{c}}$ & $92^{\mathrm{a}}$ & $68^{\mathrm{c}}$ \\
$\mathrm{T}_{3 \mathrm{ME}}$ & $91^{\mathrm{a}}$ & $84^{\mathrm{a}}$ & $71^{\mathrm{b}}$ & $75^{\mathrm{b}}$ & $93^{\mathrm{a}}$ & $80^{\mathrm{b}}$ \\
$\mathrm{T}_{4 \mathrm{ME}}$ & $92^{\mathrm{a}}$ & $85^{\mathrm{a}}$ & $83^{\mathrm{a}}$ & $87^{\mathrm{a}}$ & $94^{\mathrm{a}}$ & $91^{\mathrm{a}}$ \\
$\mathrm{LSD}$ & 16.722 & 16.62 & 12.74 & 14.174 & 15.529 & 18.34 \\
F-value & $28.64^{*}$ & $21.18^{*}$ & $11.39^{*}$ & $12.67^{*}$ & $23.73^{*}$ & $14.75^{*}$ \\
\hline
\end{tabular}

Means followed by different letters within one column differ significantly at $(P<5 \%)$

* Significant at $(P<1 \%)$ 
Table 14. Allelopathic effect of $R$. stricta leaf methanolic extract (ME) on germination percentage (\%) of test species on soil

\begin{tabular}{c|c|c|c|c|c|c}
\hline \multirow{2}{*}{ Treatments } & \multicolumn{7}{|c}{ Test species } \\
\cline { 2 - 7 } & T. aestivum & A. fatua & R. dentatus & P. minor & E. helioscopia & C. album \\
\hline $\mathrm{T}_{1 \mathrm{ME}}$ & $89^{\mathrm{a}}$ & $82^{\mathrm{a}}$ & $49^{\mathrm{c}}$ & $50^{\mathrm{d}}$ & $91^{\mathrm{a}}$ & $49^{\mathrm{d}}$ \\
$\mathrm{T}_{2 \mathrm{ME}}$ & $91^{\mathrm{a}}$ & $84^{\mathrm{a}}$ & $56^{\mathrm{c}}$ & $61^{\mathrm{c}}$ & $93^{\mathrm{a}}$ & $64^{\mathrm{c}}$ \\
$\mathrm{T}_{3 \mathrm{ME}}$ & $92^{\mathrm{a}}$ & $85^{\mathrm{a}}$ & $68^{\mathrm{b}}$ & $71^{\mathrm{b}}$ & $94^{\mathrm{a}}$ & $75^{\mathrm{b}}$ \\
$\mathrm{T}_{4 \mathrm{ME}}$ & $93^{\mathrm{a}}$ & $86^{\mathrm{a}}$ & $84^{\mathrm{a}}$ & $88^{\mathrm{a}}$ & $95^{\mathrm{a}}$ & $92^{\mathrm{a}}$ \\
$\mathrm{LSD}$ & 13.588 & 17.910 & 15.07 & 13.844 & 15.480 & 15.874 \\
F-value & $13.93^{*}$ & $22.96^{*}$ & $37.71^{*}$ & $22.94^{*}$ & $54.38^{*}$ & $12.98^{*}$ \\
\hline
\end{tabular}

Means followed by different letters within one column differ significantly at $(P<5 \%)$

*Significant at $(P<1 \%)$

\section{Radical length}

The methanolic extract significantly inhibited radical length for $R$. dentatus (45\%) and A. fatua (44\%) on filter paper, whereas, no significant effect was noted for $T$. aestivum P. minor, E. helioscopia and $C$. album showing resistance against extract. Similarly, the applications of extract into soil significantly suppressed radical length of R. dentatus $(51 \%)$ and A. fatua (50\%) as compared to control. The maximum $(96 \%)$ radical length was observed for T. aestivum P. minor, E. helioscopia and C. album (Tables 15 and 16). The final data concluded that minimum radical length was noted for R. dentatus i.e. $55 \%$ and $49 \%$ on filter paper and soil, respectively (Fig. 3b).

Table 15. Allelopathic effect of $R$. stricta methanolic extract $(M E)$ on radical length $(\mathrm{cm})$ of test species on filter paper

\begin{tabular}{c|c|c|c|c|c|c}
\hline \multirow{2}{*}{ Treatments } & \multicolumn{6}{|c}{ Test species } \\
\cline { 2 - 7 } & T. aestivum & A. fatua & R. dentatus & P. minor & E. helioscopia & C. album \\
\hline $\mathrm{T}_{1 \mathrm{ME}}$ & $9.16^{\mathrm{a}}$ & $4.81^{\mathrm{c}}$ & $5.11^{\mathrm{c}}$ & $8.14^{\mathrm{a}}$ & $9.19^{\mathrm{a}}$ & $8.14^{\mathrm{a}}$ \\
$\mathrm{T}_{2 \mathrm{ME}}$ & $9.22^{\mathrm{a}}$ & $7.31^{\mathrm{b}}$ & $7.01^{\mathrm{b}}$ & $8.22^{\mathrm{a}}$ & $9.36^{\mathrm{a}}$ & $8.21^{\mathrm{a}}$ \\
$\mathrm{T}_{3 \mathrm{ME}}$ & $9.29^{\mathrm{a}}$ & $7.25^{\mathrm{b}}$ & $7.85^{\mathrm{b}}$ & $8.39^{\mathrm{a}}$ & $9.40^{\mathrm{a}}$ & $8.27^{\mathrm{a}}$ \\
$\mathrm{T}_{4 \mathrm{ME}}$ & $9.31^{\mathrm{a}}$ & $8.64^{\mathrm{a}}$ & $9.21^{\mathrm{a}}$ & $8.41^{\mathrm{a}}$ & $9.56^{\mathrm{a}}$ & $8.32^{\mathrm{a}}$ \\
$\mathrm{LSD}$ & 3.1490 & 0.8763 & 1.7841 & 1.2929 & 0.8953 & 1.340 \\
F-value & $22.59^{*}$ & $129.81^{*}$ & $34.17^{*}$ & $66.06^{*}$ & $50.17^{*}$ & $42.47^{*}$ \\
\hline
\end{tabular}

Means followed by different letters within one column differ significantly at $(P<5 \%)$

* Significant at $(P<1 \%)$

Table 16. Allelopathic effect of $R$. stricta methanolic extract $(M E)$ on radical length $(\mathrm{cm})$ of test species on soil

\begin{tabular}{c|c|c|c|c|c|c}
\hline \multirow{2}{*}{ Treatments } & \multicolumn{6}{|c}{ Test species } \\
\cline { 2 - 7 } & T. aestivum & A. fatua & R. dentatus & P. minor & E. helioscopia & C. album \\
\hline $\mathrm{T}_{1 \mathrm{ME}}$ & $9.26^{\mathrm{a}}$ & $4.52^{\mathrm{c}}$ & $4.58^{\mathrm{c}}$ & $8.39^{\mathrm{a}}$ & $9.48^{\mathrm{a}}$ & $8.15^{\mathrm{a}}$ \\
$\mathrm{T}_{2 \mathrm{ME}}$ & $9.46^{\mathrm{a}}$ & $6.34^{\mathrm{b}}$ & $6.87^{\mathrm{b}}$ & $8.48^{\mathrm{a}}$ & $9.52^{\mathrm{a}}$ & $8.35^{\mathrm{a}}$ \\
$\mathrm{T}_{3 \mathrm{ME}}$ & $9.52^{\mathrm{a}}$ & $6.61^{\mathrm{b}}$ & $7.16^{\mathrm{b}}$ & $8.56^{\mathrm{a}}$ & $9.69^{\mathrm{a}}$ & $8.43^{\mathrm{a}}$ \\
$\mathrm{T}_{4 \mathrm{ME}}$ & $9.58^{\mathrm{a}}$ & $8.99^{\mathrm{a}}$ & $9.40^{\mathrm{a}}$ & $8.61^{\mathrm{a}}$ & $9.70^{\mathrm{a}}$ & $8.66^{\mathrm{a}}$ \\
$\mathrm{LSD}$ & 1.8406 & 1.3918 & 1.9912 & 1.5634 & 0.3063 & 1.4662 \\
F-value & $51.46^{*}$ & $97.97^{*}$ & $84.01^{*}$ & $76.89^{*}$ & $125.70^{*}$ & $93.12^{*}$ \\
\hline
\end{tabular}

Means followed by different letters within one column differ significantly at $(P<5 \%)$

* Significant at $(P<1 \%)$ 


\section{Plumule length}

Analysis of the data revealed that $R$. stricta methanolic extract significantly suppressed the plumule length of $T$. aestivum (55\%), A. fatua (33\%) and $R$. dentatus (28\%) as compared control on filter paper. Interestingly, there was no significant effect on plumule elongation of $P$. minor, E. helioscopia and $C$. album. Likewise, R. stricta methanolic extract significantly inhibited plumule length of T. aestivum (58\%), A. fatua (35\%) and $R$. dentatus (34\%) in soil. The statistical data concluded that minimum plumule length was noted for T. aestivum i.e. $45 \%$ and $42 \%$ on filter paper and soil, respectively (Tables 17 and 18). The statistical figures also proposed that maximum plumule length (96\%) was noted for P. minor, E. helioscopia and C. album (Fig. 3c).

Table 17. Allelopathic effect of R. stricta methanolic extract (ME) on plumule length (cm) of test species on filter paper

\begin{tabular}{c|c|c|c|c|c|c}
\hline \multirow{2}{*}{ Treatments } & \multicolumn{6}{|c}{ Test species } \\
\cline { 2 - 7 } & T. aestivum & A. fatua & R. dentatus & P. minor & E. helioscopia & C. album \\
\hline $\mathrm{T}_{1 \mathrm{ME}}$ & $4.15^{\mathrm{d}}$ & $5.68^{\mathrm{c}}$ & $6.81^{\mathrm{c}}$ & $7.32^{\mathrm{a}}$ & $9.06^{\mathrm{a}}$ & $8.12^{\mathrm{a}}$ \\
$\mathrm{T}_{2 \mathrm{ME}}$ & $6.52^{\mathrm{c}}$ & $6.20^{\mathrm{c}}$ & $7.05^{\mathrm{c}}$ & $7.48^{\mathrm{a}}$ & $9.09^{\mathrm{a}}$ & $8.21^{\mathrm{a}}$ \\
$\mathrm{T}_{3 \mathrm{ME}}$ & $8.14^{\mathrm{b}}$ & $7.31^{\mathrm{b}}$ & $8.16^{\mathrm{b}}$ & $7.59^{\mathrm{a}}$ & $9.13^{\mathrm{a}}$ & $8.23^{\mathrm{a}}$ \\
$\mathrm{T}_{4 \mathrm{ME}}$ & $9.28^{\mathrm{a}}$ & $8.42^{\mathrm{a}}$ & $9.52^{\mathrm{a}}$ & $7.62^{\mathrm{a}}$ & $9.16^{\mathrm{a}}$ & $8.29^{\mathrm{a}}$ \\
$\mathrm{LSD}$ & 6.4846 & 1.4971 & 0.9240 & 0.8728 & 0.2182 & 1.4428 \\
F-value & $5.43^{* *}$ & $44.74^{*}$ & $55.31^{*}$ & $55.01^{*}$ & $14.13^{*}$ & $28.95^{*}$ \\
\hline
\end{tabular}

Means followed by different letters within one column differ significantly at $(P<5 \%)$

*Significant at $(P<1 \%)$

Table 18. Allelopathic effect of $R$. stricta methanolic extract $(M E)$ on plumule length $(\mathrm{cm})$ of test species on soil

\begin{tabular}{c|c|c|c|c|c|c}
\hline \multirow{2}{*}{ Treatments } & \multicolumn{6}{|c}{ Test species } \\
\cline { 2 - 7 } & T. aestivum & A. fatua & R. dentatus & P. minor & E. helioscopia & C. album \\
\hline $\mathrm{T}_{1 \mathrm{ME}}$ & $4.01^{\mathrm{c}}$ & $5.72^{\mathrm{c}}$ & $6.59^{\mathrm{c}}$ & $7.51^{\mathrm{a}}$ & $9.24^{\mathrm{a}}$ & $8.21^{\mathrm{a}}$ \\
$\mathrm{T}_{2 \mathrm{ME}}$ & $6.13^{\mathrm{b}}$ & $5.90^{\mathrm{c}}$ & $6.72^{\mathrm{c}}$ & $7.65^{\mathrm{a}}$ & $9.33^{\mathrm{a}}$ & $8.42^{\mathrm{a}}$ \\
$\mathrm{T}_{3 \mathrm{ME}}$ & $7.04^{\mathrm{b}}$ & $7.01^{\mathrm{b}}$ & $8.01^{\mathrm{b}}$ & $7.79^{\mathrm{a}}$ & $9.56^{\mathrm{a}}$ & $8.51^{\mathrm{a}}$ \\
$\mathrm{T}_{4 \mathrm{ME}}$ & $9.58^{\mathrm{a}}$ & $8.77^{\mathrm{a}}$ & $9.94^{\mathrm{a}}$ & $7.89^{\mathrm{a}}$ & $9.61^{\mathrm{a}}$ & $8.71^{\mathrm{a}}$ \\
$\mathrm{LSD}$ & 3.7946 & 2.2971 & 1.9640 & 2.8718 & 4.0182 & 0.9841 \\
F-value & $9.34^{* *}$ & $41.64^{*}$ & $53.41^{*}$ & $58.71^{*}$ & $16.31^{*}$ & $246.78^{*}$ \\
\hline
\end{tabular}

Means followed by different letters within one column differ significantly at $(P<5 \%)$

* Significant at $(P<1 \%)$

\section{Discussion}

This study was aimed at investigating allelopathic potential of $R$. stricta by using various solvent (i.e. aqueous, methanolic, hexane) leaf extracts against selected noxious weeds of wheat crop. The germination percentage, radicle length and plumule length were observed. The results revealed that all plant extracts showed significant allelopathic activity, however, the same was varied from species to species as well as solvents. Overall, methanolic extract showed the highest degree of seed germination 
percentage inhibition, radical and plumule length for $C$. album, $P$. minor and $R$. dentatus.

The results revealed that $R$. stricta aqueous extract significantly inhibited germination percentage of $C$. album, R. dentatus and P. minor (Tables 1 and 2). The results revealed that germination percentage reduction of the $C$. album, $R$. dentatus and P. minor was concentration dependent (Fig. 1a). As far as radical length is concerned, the aqueous extract had significantly affected radical length for $R$. dentatus and A. fatua (Tables 3 and 4). The results revealed that allelopathic inhibitory effect was concentration dependent for $R$. dentatus and A. fatua (Fig. 1b). The current study indicated that aqueous extract of $R$. stricta significantly inhibited the plumule length of $A$. fatua and $R$. dentatus as compared to control, on filter paper as well as on soil (Tables 5 and 6). The results agree with that of Khan et al. (2011) who reported germination inhibition of Zea mays by stem aqueous extracts of $R$. stricta, while plumule and radical growth was significantly decreased by aqueous leave extract. Like the present investigation, the later study also revealed that the inhibitory effects were directly proportional to the extract concentration. In another study, leaf extracts of $R$. stricta significantly inhibited germination percentage and decreased plumule and radical length of seedlings of Vicia faba, Hordeum vulgare and Triticum aestivum (Ebid, 2016). Aqueous extracts from the leaves of $R$. stricta significantly reduced germination and growth of Salsola villosa by hampering functioning of the membrane and photosynthetic capacity due to reduced synthesis of chlorophyll pigments (Alqarawi et al., 2018).

$R$. stricta leaf aqueous extract owed to some toxic factors of the extract. Noteworthy, there was variation among the test treatments of different concentrations of $R$. stricta extract and control. There was significant morphological variation in the radical and plumule elongation of the tested weeds that may be attributed due to the presence of various allelochemicals in plant extract. Allelopathic interaction more effectively inhibited growth on soil in contrast to filter paper (Hegazy and Fadl-Allah, 1995; ElKhatib, 2000). These findings agreed with those of Migahid and El-khazan (2002), who studied the allelopathic effect of Zygophyllum aqueous extract on sesame and corn.

This study exhibited the allelopathic activity of various solvents on the germination and growth parameters of selected weeds. Overall, the activity of $R$. stricta extracts was found concentration dependent. Wardle et al. (1992) reported a concentration dependent inhibition of radical, plumule growth and seed germination for some weeds by $R$. stricta extract. Besides, Alam (1990) and Bora et al. (1999) also observed concentration dependent inhibition of plumule length by $R$. stricta extract. Another study revealed that there was significant reduction in germination, radical and plumule length of $Z$. mays by $R$. stricta extract (Zackrisson and Nilsson, 1992). El-khawas and Shehata (2005) stated that the inhibitory effect of allelochemicals on seed growth due to changes in enzyme activities that affect the transport of storage substances.

Our results revealed that $R$. stricta methanolic leaf extract imposed significant inhibitory effects on the germination and growth parameters of all the tests weeds. The findings of current study confirmed the earlier findings on cowpea and rice plumule, radicle length and germination that were significantly checked with increasing concentration of $R$. stricta methanolic leaf extract (Jadhar and Gayanar, 1992). Moreover, seed germination and seedling growth of different crops and weed had been significantly checked by inhibitory potential of $R$. stricta extracts (Lisanework and Michelson, 1993; Khan et al., 2008). 
Suppressive effect on plumule length and seed germination was also described by Tefera (2002) and Siddiqui et al. (2009). These findings were in accordance to previous studies who stated that $R$. stricta leaf extract reduced growth of weeds species (Maciel et al., 2003; Kaul and Bansal, 2002). Germination response of various field crops varies in the presence of $R$. stricta leaf extract that confirms our observation of the differences in germination response of tested species. Several studies demonstrated the allelopathic potential of Parthenium hysterophorus (Tawaha and Turk, 2003), Brassica nigra (Singh et al., 2003) and Raphanus raphanistrum (Batish et al., 2002).

The leaf extracts were more toxic and more allelopathic in nature owing to relatively higher inhibitory potential in contrast to other plant parts (Hussain and Gadoon, 1981; Norsworthy, 2003). Similar allelopathic effects were reported from other plants (Inderjit and Duke, 2003; Hussain et al., 2011).

\section{Conclusion}

This study was aimed at evaluation of allelopathic potential of $R$. stricta against some noxious weeds of wheat crop by using aqueous, hexane and methanolic extracts on filter paper and soil. The results revealed that methanolic extract highly reduced seed germination percentage of $C$. album, $P$. minor and $R$. dentatus. The same extract caused significant radical and plumule length reduction of $R$. dentatus and A. fatua. Based on the results, it can be concluded that $R$. stricta showed good allelopathic activity that may be used in future herbicide screening program prior to launch in the market.

Acknowledgements. This paper is extracted from the $\mathrm{PhD}$ thesis of the first author.

\section{REFERENCES}

[1] Alam, S. M. (1990): Effect of wheat straw extract on germination and seedling growth of wheat (cv. Pavon). - Wheat Informative Service 71: 16-18.

[2] Alqarawi, A. A., Hashem, A., Kumar, A., Al-Arjani, A. B. F., Abd-Allah, E. F., Dar, B. A., Egamberdieva, D. (2018): Allelopathic effects of the aqueous extract of Rhazya stricta on growth and metabolism of Salsola villosa. - Plant Biosystematics DOI: 10.1080/11263504.2018.1439117.

[3] Anwar, T., Khalid, S., Arafat, Y., Sadia, S., Riaz, S. (2013): Allelopathic suppression of Avena fatua and Rumex dentatus in associated crops. - Pakistan Journal of Weed Science Research 19: 31-43.

[4] Anwar, T., Khalid, S., Saeed, M., Mazhar, R.., Qureshi, H., Rashid, M. (2016): Allelopathic interference of leaf powder and aqueous extracts of hostile weed: Parthenium hysterophorus (Asteraceae). - Science International 4: 86-93.

[5] Anwar, T., Khalid, S., Mazhar, R., Qureshi, H., Rashid, M. (2017a): Herbicidal potential of selected species to overcome weed infestation in Triticum aestivum, Zea mays and Helianthus annuus. - Pakistan Journal of Weed Science Research 23: 49-63.

[6] Anwar, T., Khalid, S., Panni, M. K., Qureshi, H., Rashid, M. (2017b): Allelopathic effect of Euphorbia helioscopia on Avena fatua, Rumex dentatus, Helianthus annuus, Zea mays and Triticum aestivum. - Pakistan Journal of Weed Science Research 23: 165-177.

[7] Arora, K., Batish, D. R., Singh, H. P., Kohli, R. K. (2015): Allelopathic potential of the essential oil of wild marigold (Tagetes minuta L.) against some invasive weeds. - Journal of Environmental \& Agricultural Sciences 3: 56-60. 
[8] Batish, D. R., Singh, H. P., Kohli, R. K., Saxena, D. B., Kaur, S. (2002): Allelopathic effect of Parthenium against two weedy species, Avena fatua and Bidens pilosa. Environmental Experimental Botany 47: 149-155.

[9] Biljana, M. B., Kragujevac, D. Z. J. (2015): Allelopathic relations of selected cereal and vegetable species during seed germination and seedling growth. - Journal of Science 37: 135-142.

[10] Bora, I. P., Singh, J., Borthakur, R., Bora, E. (1999): Allelopathic effect of leaf extracts of Acacia auriculiformis on seed germination of some agricultural crops. - Annals Forestry 7: 143-146.

[11] Chon, S. U., Jang, H. G., Kim, D. K., Kim, Y. M., Boo, H. O., Kim, Y. J. (2005): Allelopathic potential in lettuce (Lactuca sativa L.) plants. - Scientia Horticulture 106(3): 309-317.

[12] Dallali, S., Rouz, S., Aichi, H., Hassine, H. B. (2017): Phenolic content and allelopathic potential of leaves and rhizosphere soil aqueous extracts of white horehound (Maribum vulgare L.). - Journal of New Sciences 39: 342-353.

[13] Dayan, F. E., Romagni, J. G., Duke, S. O. (2000): Investigating the mode of action of natural phytotoxins. - Journal of Chemical Ecology 20: 2079-2093.

[14] Delcour, I., Spanoghe, P., Uyttendaele, M. (2015): Literature Review: Impact of climate change on pesticide use. - Food Research International 68: 7-15.

[15] Ebid, A. I. (2016): Allelopathic effect of three wild species on seed germination and seedling growth of Vicia faba, Hordeum vulgare and Triticum aestivum. - Journal Agricultural Ecology Research International 6(1): 1-7.

[16] El-Khatib, A. A. (2000): The ecological significance of allelopathy in the community organization of allhagrae-corum. - Biology Plantarum 43: 42-431.

[17] El-Khawas, S. A., Shehata, M. M. (2005): The allelopathic potentialities of Acacia nilotica and Eucalyptus rostrata on monocot (Zea mays L.) and dicot (Phaseolus vulgaris L). - Plant Biotechnology 4: 23-34.

[18] Hegazy, A. K., Fadl-Allah, E. M. (1995): Inhibition of seed germination and seedling growth by Cleome droserifolia and allelopathic effect on fungi. - Egypt Journal Arid Environment 29: 3-13.

[19] Hussain, F., Gadoon, M. A. (1981): Allelopathic effects of Sorghum vulgare Pers. Oecologia 51: 284-288.

[20] Hussain, M. I., Gonzalez, L., Reigosa, M. J. (2011): Allelopathic potential of Acacia melanoxylon on the germination and root growth of native species. - Weed Biology Management 11: 18-28.

[21] Inderjit, Duke, S. O. (2003): Eco-physiological aspects of allelopathy. - Planta 217: 529539.

[22] Jadhar, B. B., Gayanar, D. G. (1992): Allelopathic effect of Acacia auriculiformis on germination of rice and cowpea. - Indian Journal Plant Physiology 1: 86-89.

[23] Kaul, S., Bansal, G. L. (2002): Allelopathic effect of Ageratina adenophora on growth and development of Lantana camara. - Indian Journal of Plant Physiology 7: 195-197.

[24] Khan, M., Hussain, F., Musharaf, S. (2011): Allelopathic effects of Rhazya stricta decne on seed germination and seedling growth of maize. - African Journal Agricultural Research 6(30): 6391-6396.

[25] Khan, M. A., Hussain. I., Ahmad, E. (2008): Allelopathic effect of Eucalyptus (Eucalyptus camaldulensis L.) on germination and seedling growth of (Triticum aestivum L.). - Pakistan Journal of Weed Science Research 14: 9-18.

[26] Lisanework, N., Michelen, A. (1993): Allelopathy in agro forestry systems. The effects of leaf extracts of Eucalyptus species on three crops. - Agro-forestry Systematics 21: 63-74.

[27] Maciel, C. D. G., Correa, M. R., Alves, E., Negrisoli, E., Velini, E. D., Rodrigues, J. D., Ono, E. O., Boaro, C. S. F. (2003): Influence of Brachiaria decumbens straw management on the initial development of Glycine max and Euphorbia heterophylla. Plant Weed 213: 635-637. 
[28] Manimegalai, A., Manikandan, T., Sheela, R., Geetha, S. (2012): Allelopathic influence of Tectona grandis leaves on the germination of black gram (Vigna mungo L.) and green gram (Vigna radiate L.). - International Journal of Current Science 12: 241-244.

[29] Migahid, M. M., El-khazan, M. M. (2002): Allelopathic potential in Zygophyllum album and its effect on growth and metabolism of some crop plants. - International Conference on Biological Sciences Tanta Uni. 2: 46-63.

[30] Nekonam, M. S., Kraimmojeni, H., Sharifnabi, B., Razmjoo, J., Amini, H., Bahrami, F. (2014): Assessment of some medicinal plants for their allelopathic potential against redroot pigweed (Amaranthus retroflexus). - Journal of Plant Protection and Research 54: 90-95.

[31] Norsworthy, J. K. (2003): Allelopathic potential of wild radish (Raphanus raphanistrum). - Weed Technology 17: 307-313.

[32] Omar, A., Almaghrabi. (2008): Allelopathic effect of Rhazya stricta leaves leachate on germination and seedlings of some plants. - Egypt Journal of Experimental Biology 4: 99-103.

[33] Qureshi, R., Waheed, A., Arshad, M. (2009): Weed communities of wheat crop in district Toba Tek Singh, Pakistan. - Pakistan Journal of Botany 41: 239-245.

[34] Sabir, S. H., Khan, E. A., Shah, H., Ahmed, N., Khan, J., Sadozai, G. U. (2016): Allelopathic sorghum water extract helps to improve yield of sunflower (Helianthus annuus L.). - Pakistan Journal of Botany 48: 1197-1202.

[35] Sahu, A., Devkota, A. (2013): Allelopathic effects of aqueous extract of leaves of Mikania micrantha H. B. K. on seed germination and seedling growth of Oryza sativa L. and Raphanus sativus L. - Science World Journal 11: 90-93.

[36] Shahid, M., Ahmad, B., Khattak, R. A., Hassan, G. Khan, H. (2006): Response of wheat and its weeds to different allelopathic plant water extracts. - Pakistan Journal of Weed Science Research 12: 61-68.

[37] Siddiqui, S., Meghvansi, M. K., Yadav, K., Yadav, R., Wani, F. A., Ahmad, A. (2009): Efficacy of aqueous extracts of five arable trees on the seed germination of Pisum sativum L. var. VRP-6 and KPM-522. - Botany Research International 2: 30-35.

[38] Singh, H. P., Batish, D. R., Pandher, J. K., Kohli, R. K. (2003): Assessment of allelopathic properties of Parthenium hysterophorus residues. - Agricultural Ecosystem and Environment 95: 537-541.

[39] Tawaha, A. M., Turk, M. A. (2003): Allelopathic effects of black mustard (Brassica nigra) on germination and growth of wild barley (Hordeum spontaneum). - Journal Agronomy and Crop Sciences 189: 298-303.

[40] Tefera, T. (2002): Allelopathic effects of Parthenium hysterophorus extracts on seed germination and seedling growth of Eragrostis tef (Zucc.) Trotter. - Journal Agronomy and Crop Sciences 188: 306-310.

[41] Thi, H. L., Lan, P. T. P., Chin, D. V., Kato-Noguchi, H. I. S. A. S. H. I. (2008): Allelopathic potential of cucumber (Cucumis sativus) on barnyard grass (Echinochloa crus-galli). - Weed Biology Management 8(2): 129-132.

[42] Wardle, D. A., Nicholson, K. S., Ahmed, M. (1992): Comparison of osmotic and allelopathic effects of grass leaf extracts on grass seed germination and radicle elongation. - Plant and Soil 140: 315-319.

[43] Zackrisson, O., Nilsson, M. C. (1992): Allelopathic effects by Empetrum hermaphroditum on seed germination of two boreal tree species. - Canadian Journal of Forest Research 22: 1310-131. 